\title{
Rentabilidad de la inversión en educación superior de los egresados de la Universidad Continental
}

\author{
Investment profitability in higher education from \\ graduates at Universidad Continental
}

Gustavo Loayza Acosta', Juan Pérez Ticse 2

Universidad Continental

\section{RESUMEN}

Objetivos: Determinar los principales factores académicos que impactan positivamente en el retorno de la educación superior universitaria de los egresados de las Escuelas Profesionales de Ingeniería Informática, Administración y Contabilidad de la Universidad Continental en el período 2006-2007. Métodos: Uso de mínimos cuadrados ordinarios (MCO) con datos panel a través de las ecuaciones mincerianas y de las funciones generatrices de ingresos, para la estimación de la rentabilidad privada de la educación. Resultados: Los factores principales que impactan positivamente en el retorno de la educación superior universitaria son los años de educación, años de experiencia del trabajador y años de experiencia expresada en horas de trabajo. Se determinó que existen diferencias en las tasas de rentabilidad educativa por sexo, el nivel de ingresos de los varones es superior al de las mujeres; también que existen diferencias salariales entre contratos part o full time, por lo que deberían usarse variables que controlen por el número total de horas trabajadas. Conclusiones: Las variables determinantes de la rentabilidad son: nivel educativo alcanzado, salarios, experiencia laboral, edad y género. La variable que afecta negativamente al salario viene a ser la experiencia laboral y las variables que afectan positivamente al salario son edad y género. Un año de educación adicional impacta negativamente a la probabilidad de participar en el mercado laboral, esto porque para una persona educada es importante continuar con la educación. Esto está en consonancia con educación como una inversión. La educación formal aumenta el salario de reserva y desalienta a las personas a trabajar. La experiencia incentiva a las personas para entrar en el mercado laboral.

Palabras Clave: Retorno en educación, mínimos cuadrados ordinarios, experiencia laboral.

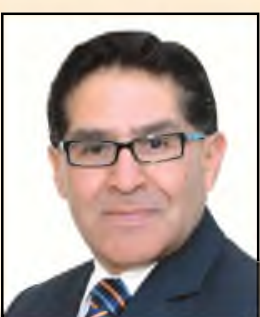

Gustavo Loayza

gloayza@continental.edu.pe

Historial del artículo: Recibido: 12 de agosto de 2014 Aprobado: 23 de noviembre de 2014 Disponible en línea: 30 de diciembre de 2014 la teoría del capital humano y su visión de la

1 Economista, Magíster en Administración, coordinador de la Escuela Académica de Economía y docente investigador de la Universidad Continental.

2 Economista, docente de la Universidad Continental. 


\section{ABSTRACT}

Objectives: To determine the main academic factors which positively impact the university education return in the Informatics Engineering, Business Management and Accounting Professional Schools' graduates from the Universidad Continental in 2006 2007. Methods: Using ordinary least squares (OLS) with panel data through Mincer equations and revenue generatrix functions, for the estimated private profitability in education. Results: The main factors that positively impact the university education return are the years of education, years of experience working and years of experience expressed in work hours. It was determined that there are differences in educational performance rates by gender, income level of men is higher than women's; also there are wage differences between part or full time contracts, so variables that control the total number of worked hours should be used. Conclusions: The profitability's determining variables are: attained education level, wages, work experience, age and gender. The variable that negatively affects wage is the work experience and the variables that positively affect wages are age and gender. A year of additional education negatively impacts the probability of labour market's participation, this because for an educated person is important to continue with education. This is in line with the human capital theory and its vision of education as an investment. Formal education increases the reservation wage and discourages people to work. Experience encourages people to enter in the labour market.

Keywords: Education returns, ordinary least squares, work experience.

\section{INTRODUCCIÓN}

La motivación central de este estudio es determinar los principales factores que impactan positivamente en el retorno de la educación superior universitaria de egresados de la Universidad Continental de las Escuelas Profesionales de Ingeniería
Informática, Administración y Contabilidad en el período 2006-2007.

Se analiza las brechas de remuneraciones e ingreso entre trabajadores de las escuelas profesionales mencionadas anteriormente. En segundo lugar, se analiza las tasas de retorno a la educación y su evolución durante los cinco años después de egresado, tanto a nivel agregado como entre grupos de profesionales de cada carrera. Finalmente, examinamos si existe evidencia de diferencias en las tasas de retorno para diversos grupos de la población; es decir, si existe una mayor heterogeneidad en el desempeño en el mercado laboral. Ello exige identificar y analizar el impacto de las variables que influyen sobre la tasa de retorno académico, en especial en el caso de aquellas Escuelas Profesionales de mayor antigüedad de la Universidad Continental como son Contabilidad, Ingeniería Informática y Administración.

Uno de los primeros economistas que ingresó a investigar este tema fue Mincer (1) quien se focaliza en la dinámica de los ingresos a lo largo del ciclo de vida y en la relación entre ingresos observados, ingresos potenciales e inversión en capital humano (tanto en términos de educación formal como de inversión en el trabajo), el autor planteó un modelo construido sobre identidades contables basado en las posturas clásicas donde encuentra una relación positiva entre el logaritmo de los ingresos con los años de educación y experiencia.

Por otro lado, Bourguignon, Ferreria, et al (2) trataron de explicar la convexificación de los retornos a la educación, es decir plantearon que los retornos a la educación están creciendo como consecuencia del proceso de apertura de nuestras economías, la globalización, el cambio tecnológico y la complementariedad del capital humano calificado con el capital físico.

Con respecto al capital humano Chiswick (3) define al capital humano como la cantidad de conocimientos técnicos y habilidades que posee la población trabajadora de un país, 
procedente de las inversiones en educación formal y en formación en el trabajo.

De acuerdo con la nueva teoría del crecimiento Angrist y Krueger (4) el aumento en productividad no es un factor exógeno sino endógeno y altamente relacionado con la acumulación de los factores de producción y los niveles de conocimientos. Lucas (5) por otra parte, afirma que los trabajadores con mayor capital humano son más productivos, independientemente de su nivel de habilidad; por tanto el capital humano se acumula mediante la producción explicita es decir, la acumulación de habilidades (experiencia). Por lo que la producción depende entonces tanto de conocimientos privados como del acervo de conocimientos agregados. Su teoría del capital humano, asume que los trabajadores adquieren habilidades que maximizan el valor presente de sus ingresos a lo largo de sus vidas.

Heckman (6) en su estudio dentro de la teoría del capital humano explica, que la inversión en recursos humanos es considerada similar a la inversión en otros recursos. Todo costo en el que se incurre es realizado bajo la expectativa de beneficios futuros superiores y por lo tanto, la inversión en la última unidad de capital humano invertirá solo si los beneficios esperados son mayores a los costos. Reveló la existencia de que existen tres costos del capital humano:

a) Costos directos; relacionados con las colegiaturas, libros, transporte y demás gastos en que se incurre al estudiar.

b) Costos de oportunidad generada por el salario que pudo haber sido adquirido al trabajar en lugar de estudiar.

c) Costo de pérdidas físicas aquellas generadas por que el aprender es difícil.

También reveló la forma de la determinación del ingreso bajo el mismo supuesto de la determinación de acumulación donde los individuos adquieren habilidades que maximizan el valor presente de sus ingresos a lo largo de sus vidas se sustentan los conceptos económicos para estudiar la determinación del ingreso.

El primer trabajo no econométrico que exploró el tema de los ingresos y concordancia ocupacional por profesiones específicas fue el de Arregui (7) quien explicó que el principal activo de los pobres es su mano de obra y escaso capital humano. Para el autor habría que acelerar la inversión en educación básica para los pobres y no quedarse allí: también habría que promover su inserción en la educación superior, la única manera de asegurar su salida permanente de la pobreza.

En el Perú, Yamada (8) propuso la correcta estimación de la ecuación de ingresos de Micer y el retorno a la educación por el método de Mínimos Cuadrados Ordinarios (MCO) dependen crucialmente del supuesto de exogeneidad de la variable "educación". Si, por el contrario, la educación fuera endógena, el estimador MCO de los retornos a la educación sería inconsistente. Se requeriría entonces de una metodología alternativa de variables instrumentales (VI) que permitan estimar de manera consistente los retornos a la educación. Supóngase, por ejemplo, que, en el modelo empírico simplificado de ingresos de Mincer: $\operatorname{Ln}(E)=\operatorname{Ln}\left(E_{-} \circ\right)+r S+e$. En el término de error (e) de la ecuación se encuentra la variable "motivación o habilidad innata», que no es observada por el econometrista en los datos. Ahora bien, el nivel de motivación o habilidad del individuo afecta tanto los años de educación (S) como el nivel de ingreso del individuo $\operatorname{Ln}(E)$. Por ello, la variable "educación» (S) ya no es exógena en la regresión y técnicamente la varianza entre ella y el error, $\mathrm{E}(\mathrm{Se})$, no es cero. Por tanto, el coeficiente de retorno a la educación no puede ser identificado correctamente con el procedimiento $\mathrm{MCO}$.

El procedimiento de variables instrumentales consiste en encontrar una variable alternativa $Z$ que cumpla con dos requisitos fundamentales: 1) que no esté correlacionada con el error (es decir que $E$ (Ze) sea cero), y 2) que esté correlacionada con la variable $S$ que va a reemplazar (es 
decir que $E$ (ZS) sea diferente de cero). En pocas palabras, se debe buscar una variable alternativa $Z$ que esté relacionada con los años de educación del individuo, pero que no afecte sus ingresos.

\section{MATERIAL Y MÉTODOS}

Investigación de tipo empírica, método científico (inductivo - deductivo) por el alcance que tendrá esta investigación en futuras investigaciones para generalizar a otras carreras profesionales.

Para esta investigación se tiene en cuenta el uso de MCO con datos panel y modelado por las ecuaciones mincerianas. Dado que este estudio se ubica dentro del tipo de investigación aplicada, cuyo análisis empírico consiste en probar mediante un modelo econométrico los principales factores o variables relacionadas con la rentabilidad de la inversión académica de los alumnos, los datos serán semestrales y anuales. Se analizó cómo cambia el impacto en sus ingresos la formación académica a lo largo de la carrera universitaria.

La población objeto de estudio corresponde a los egresados de la Universidad Continental de los años 2006-2007 de las escuelas profesionales de Ingeniería Informática, Contabilidad y Administración, con un total de 385 egresados, la muestra fue de 214, de manera representativa. Para la muestra se utilizó la fórmula para poblaciones finitas y se realizó el uso de muestreo aleatorio simple.

$$
n=\frac{z^{2} \times p \times q \times N}{e^{2} \times(N-1)+z^{2} \times p \times q}
$$

Donde:

$\mathrm{n}$ = Tamaño de la muestra

$z$ = Nivel de confianza dado en desviación estandar $95 \%=1,96$

$\mathrm{p}=$ Probabilidad que el evento ocurra

$q=$ Probabilidad que el evento no ocurra

$N$ = Población bajo estudio

e $=$ Error de estimación (en $5 \%$ )
Para la construcción de la base de datos, se utilizó la data estadística generada del sistema de seguimiento al egresado de la Oficina de Oportunidades Laborales de la Universidad Continental.

El alcance central de este estudio es analizar la evolución de las remuneraciones y tasas de retorno de las escuelas profesionales de Contabilidad, Ingeniería. Informática y Administración con estudios en la Universidad Continental. En primer lugar, analizamos las brechas de remuneraciones e ingreso entre los egresados de las escuelas profesionales mencionadas anteriormente.

\section{RESULTADOS}

En el análisis de la tasa de retorno de la educación, la estimación de las llamadas ecuaciones Mincerianas es una referencia obligada. El modelo de interpretación que formulamos es el siguiente:

$\ln \left(\right.$ salario $\left._{\mathrm{ij}}\right)=$

$\beta_{0}+\beta_{2} \operatorname{Educ}_{\mathrm{ij}}+\beta_{3} \operatorname{Exp}_{\mathrm{ij}}+\beta_{4} \operatorname{Exp}_{\mathrm{ii}}^{2}+\varepsilon_{\mathrm{ij}}$

Donde:

$\ln \left(\right.$ salario $\left._{\mathrm{ij}}\right)=$ Es el incremento porcentual del salario percibido por los estudiantes de la Universidad Continental

Educ $=\quad$ Es los años de experiencia y preparación académica

$\operatorname{Exp}=\quad$ Es las horas de trabajo que

$\operatorname{Exp}^{2}=\quad \begin{aligned} & \text { los estudiantes realizaron } \\ & \text { cuadrado de las horas }\end{aligned}$ trabajadas

$\varepsilon \quad=\quad$ perturbación aleatoria

$\ln \left(\right.$ salario $\left._{\mathrm{ij}}\right)=7,66-0,07 \times$ Educ $_{\mathrm{ij}}-0,12$

Tabla $N^{\circ}$ 1: Tamaño de la población y muestra del estudio

\begin{tabular}{lrr}
\hline $\begin{array}{l}\text { Egresados 2006 } \\
\text { al 2007 }\end{array}$ & Población & Muestra \\
\hline Ingeniería Informática & 220 & 130 \\
Administración & 95 & 45 \\
Contabilidad & 70 & 39 \\
\hline
\end{tabular}


$x \operatorname{Exp}_{\mathrm{ii}}+0,01 \operatorname{Exp}^{2}{ }_{\mathrm{ij}} \quad \ldots$ (1)

En la tabla $\mathrm{N}^{\circ} 2$, que contiene la primera Ecuación Minceriana observamos que presenta un $r^{\wedge} 2$ ( $r$ cuadrado) de 0,0369 es decir sólo el $3 \%$ de los datos observados explican el comportamiento incremental de los salarios, por tanto para este caso globalmente no estamos frente a una buena estimación. Pero si tomamos en cuenta los $T$ estadísticos encontramos que los años de experiencia de alguna forma alcanza a ser válida para nuestro modelo con un $\dagger$ estadístico de $-2,41$, cuya probabilidad es de $0,017, y$ es menor que el p-value, de que la variable educación que acumulan los estudiantes este correlacionado con los ingresos de sus padres ya que eso depende el pago de sus estudios y los años de educación acumulados.

La figura $N^{0} 1$, que muestra la relación de la variable educación con respecto al log (salarios), evidencia una relación positiva entre la educación y los salarios recibidos por los egresados. Por lo tanto, a mayor años de educación corresponde mayor nivel de salarios. Lo cual coincide con la evidencia empírica.

Para mejorar el modelo econométrico de

Tabla N ${ }^{\circ}$ 2: Primera Ecuación Minceriana

\begin{tabular}{|c|c|c|c|c|c|c|c|}
\hline \multicolumn{8}{|c|}{ Reg. Isalario educ exper exper2 } \\
\hline Source & SS & $d f$ & \multicolumn{2}{|c|}{ MS } & & \multirow{2}{*}{$\begin{array}{l}\text { Number of obs } \\
F(3,210)\end{array}$} & \multirow{2}{*}{$\begin{array}{l}=214,00000 \\
=2,68000\end{array}$} \\
\hline Model & 17,7468180 & 3 & \multicolumn{2}{|c|}{0,591560601} & & & \\
\hline Residual & 46,3437861 & 210 & \multicolumn{2}{|c|}{0,220684696} & & Prob $>F$ & $=0,04790$ \\
\hline \multirow[t]{3}{*}{ TOTAL } & 48,1184679 & 213 & \multicolumn{2}{|c|}{0,225908300} & & R-squared & $=0,03690$ \\
\hline & & & & & & Adj R-squared & $=0,02310$ \\
\hline & & & & & & Root MSE & $=0,46977$ \\
\hline Isalario & Coef. & Std & Err. & $t$ & $P>(t)$ & (95\% Conf. & Interval) \\
\hline educ & 0,7184900 & 0,0 & 88796 & 1,18 & 0,239 & $-0,4816440$ & 0,1918625 \\
\hline exper & $-0,1194932$ & 0,0 & 96764 & $-2,41$ & 0,017 & $-0,2174216$ & $-0,0215648$ \\
\hline expe2 & 0,0068060 & 0,00 & 35316 & 1,93 & 0,055 & $-0,0001559$ & 0,0137679 \\
\hline cons & 7,6635240 & 0,3 & 21349 & 21,16 & 0,000 & 6,9496380 & 8,3774090 \\
\hline
\end{tabular}

la misma forma las horas de trabajo que realizan los egresados se encuentra con $\dagger$ estadístico de 1,93 para una probabilidad de 0,055 . En consideración a esto resulta relevante para nuestro modelo las variables de años de experiencia y las horas de trabajo. Ante un estadístico $f$ con 3 grados de libertad es decir por la obtención de una varianza más ajustada, se obtiene un 2,68 relativamente aceptable.

Por tanto, econométricamente estos resultados se debe posiblemente a la omisión de variables relevantes que puedan explicar los salarios de las escuelas profesionales de Ingeniería Informática, Contabilidad y Administración se sospecha nuestro estudio, recurrimos a la estimación de la segunda ecuación.

$$
\begin{aligned}
& \operatorname{Ln}(\text { salario })_{\mathrm{ij}}= \\
& \quad \beta_{0}+\beta_{1} \text { exper }+\beta_{2} \text { periodo }+\beta_{3} \text { carrera }+ \\
& \beta_{4} \text { educ }+\beta_{5} \text { género }+\varepsilon_{\mathrm{ij}} \ldots \text { (2) }
\end{aligned}
$$

Donde:

$$
\begin{array}{ll}
\operatorname{Ln}(\text { salario)ii = } & \text { sigue siendo el incremento } \\
& \text { porcentual del salario } \\
= & \text { son los años de experiencia } \\
\text { exper } & \text { se refiere al periodo } \\
\text { periodo } & \text { semestral que egresó } \\
= & \text { referido al tipo de carrera } \\
\text { carrera } & \text { profesional que eligió } \\
= & \text { nos indica en que edad se }
\end{array}
$$




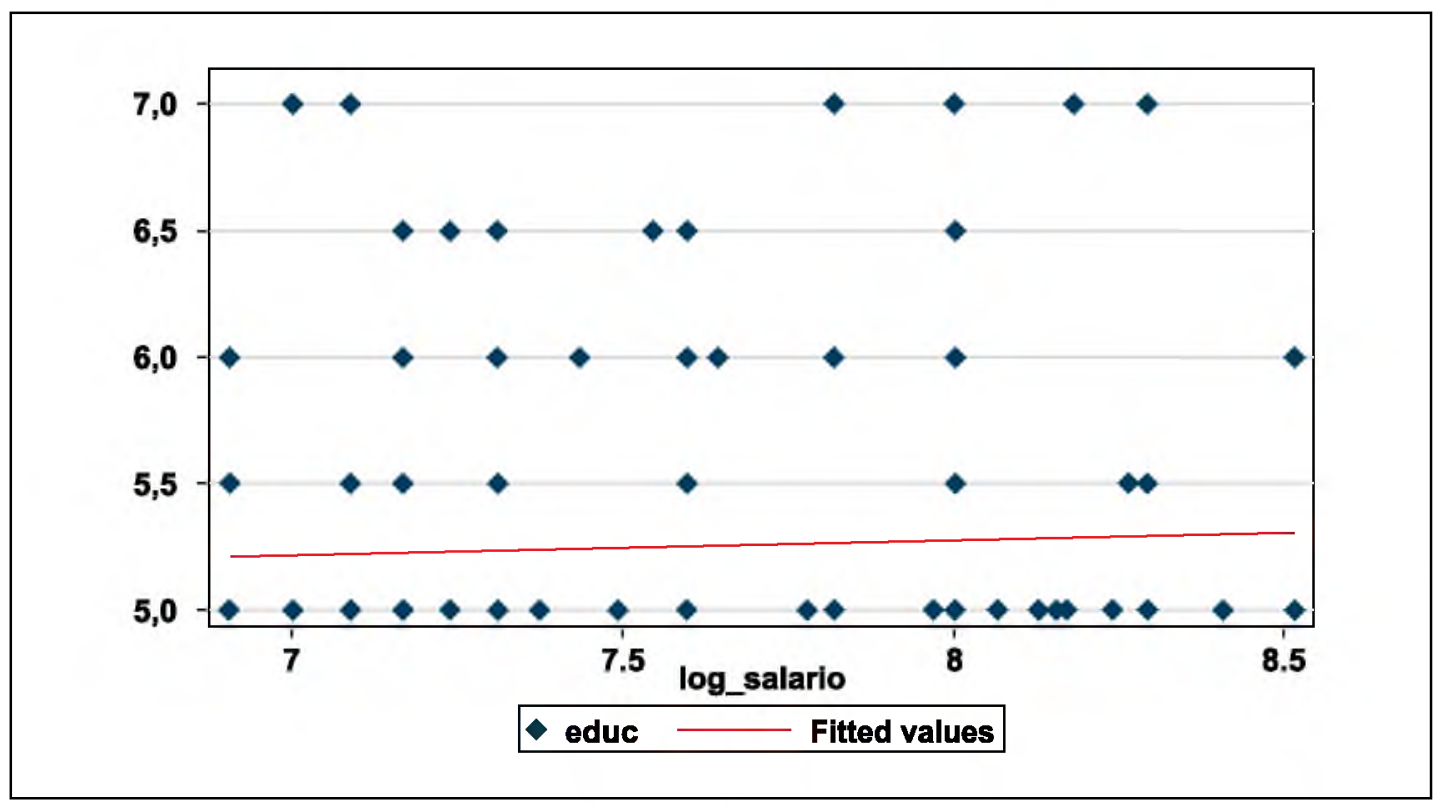

Figura $N^{\circ} 1$ : Relación entre educación y salario (log_salario) recibido por los egresados.

Tabla N ${ }^{\circ}$ 3: Mejor modelo estimado

\begin{tabular}{|c|c|c|c|c|c|c|c|}
\hline \multicolumn{8}{|c|}{ Reg. logsalario exper } \\
\hline Source & SS & $d f$ & \multicolumn{2}{|c|}{ MS } & & Number of obs & $=212,00000$ \\
\hline Model & 14,5683704 & 1 & \multicolumn{2}{|c|}{14,568370400} & & $F(3,210)$ & $=78,87000$ \\
\hline Residual & 38,78743437861 & 210 & \multicolumn{2}{|c|}{0,184702165} & & Prob $>F$ & $=0,00000$ \\
\hline \multirow[t]{3}{*}{ TOTAL } & \multirow[t]{3}{*}{53,3558249} & 211 & \multicolumn{2}{|c|}{0,252871208} & & R-squared & 0,27300 \\
\hline & & & & & & Adj R-squared & $=0,26960$ \\
\hline & & & & & & Root MSE & $=0,42977$ \\
\hline logsalario & Coef. & Std & Err. & $t$ & $P>(t)$ & (95\% Conf. & Interval) \\
\hline exper & $-0,1361178$ & 0,0 & 53266 & $-8,88$ & 0,000 & $-0,1663315$ & $-0,1059041$ \\
\hline _cons & 1,3324980 & 0,11 & 44859 & 12,75 & 0,000 & 1,1265220 & 1,5384730 \\
\hline
\end{tabular}

encuentra el egresado

género $=$ referido al sexo del egresado

$\operatorname{Ln}($ salario) $=1,332498-0,1361178 x$ exper

En la tabla $\mathrm{N}^{\circ} 3$, se observa el mejor modelo estimado con un r2 de 0,2730 y un f estadístico de 78,87 con una probabilidad de 0,000 y un grado de libertad, esto se debe a el ajuste de la varianza para obtener una disminución de los errores de la estimación, en este modelo la variable experiencia resulta ser significativo dado que la probabilidad es de 0,00 por tanto la experiencia nos indicaría que a medida
Tabla $N^{\circ}$ 4: Relación de años de estudio con la media salarial

\begin{tabular}{rr}
\hline \multicolumn{2}{c}{ Table educ, c (mean salario) } \\
Población & mean(salario) \\
\hline 5 & 2162,43 \\
5,5 & 2352,11 \\
6 & 2133,33 \\
6,5 & 2010 \\
7 & 2797,5 \\
\hline
\end{tabular}

que el egresado va acumulando mejoras en su productividad haciendo que se eleve sus mejoras sobres sus salarios a medida 


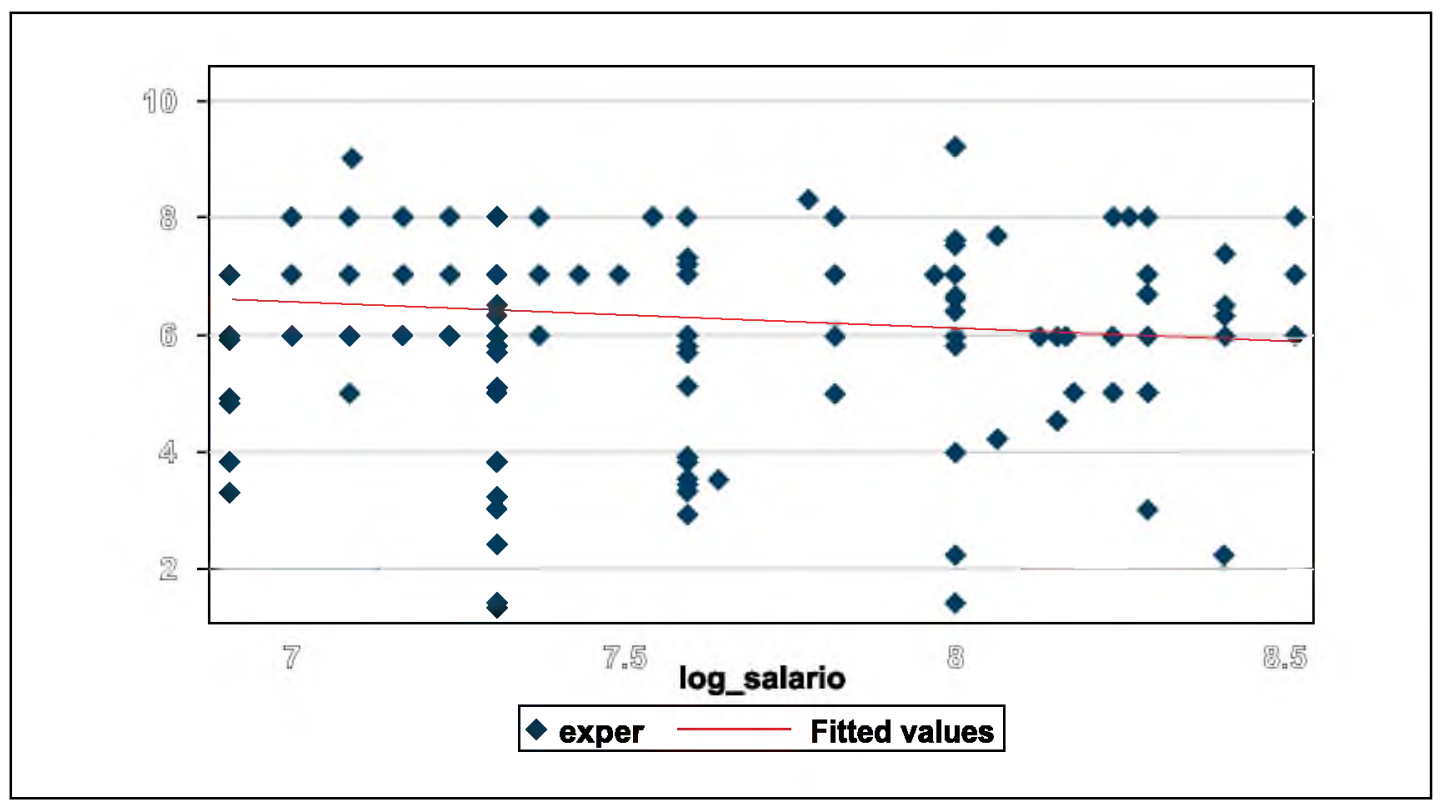

Figura $N^{\circ}$ 2: Relación de salario (log Salario) versus años de experiencia

que los ascensos se formalizan a partir de la experiencia laboral.

En la figura $\mathrm{N}^{\circ} 2$, se muestra la relación de los montos de los salarios versus los años de experiencia. Como vemos existe una relación inversa entre las dos variables. Esto debido a la variabilidad de los salarios de los alumnos. Toda vez que algunos tienen salarios muy altos y por otro lado algunos no trabajan. Revisando la variabilidad por escuelas profesionales la que tiene mayor variabilidad es la escuela de Administración y con menor variabilidad la escuela de Contabilidad.

La tabla $\mathrm{N}^{\circ} 4$, que muestra el resultado de esta nueva ecuación tenemos el promedio de salarios por años de educación donde puede notarse que a más años de educación mayor nivel de ingresos excepto en algunos egresados que son pocos que

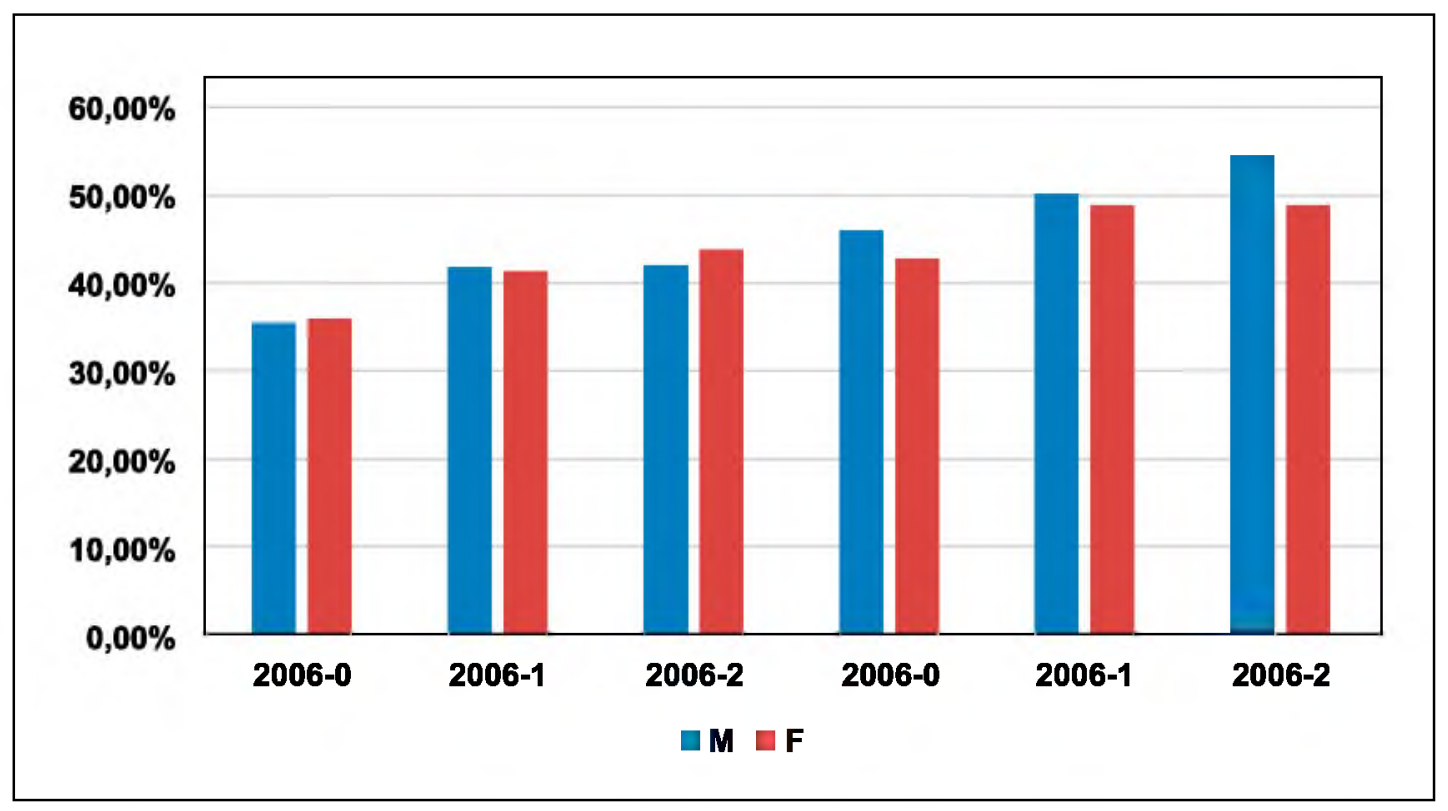

Figura $\mathrm{N}^{\circ}$ 3: Incremento salarial por género 


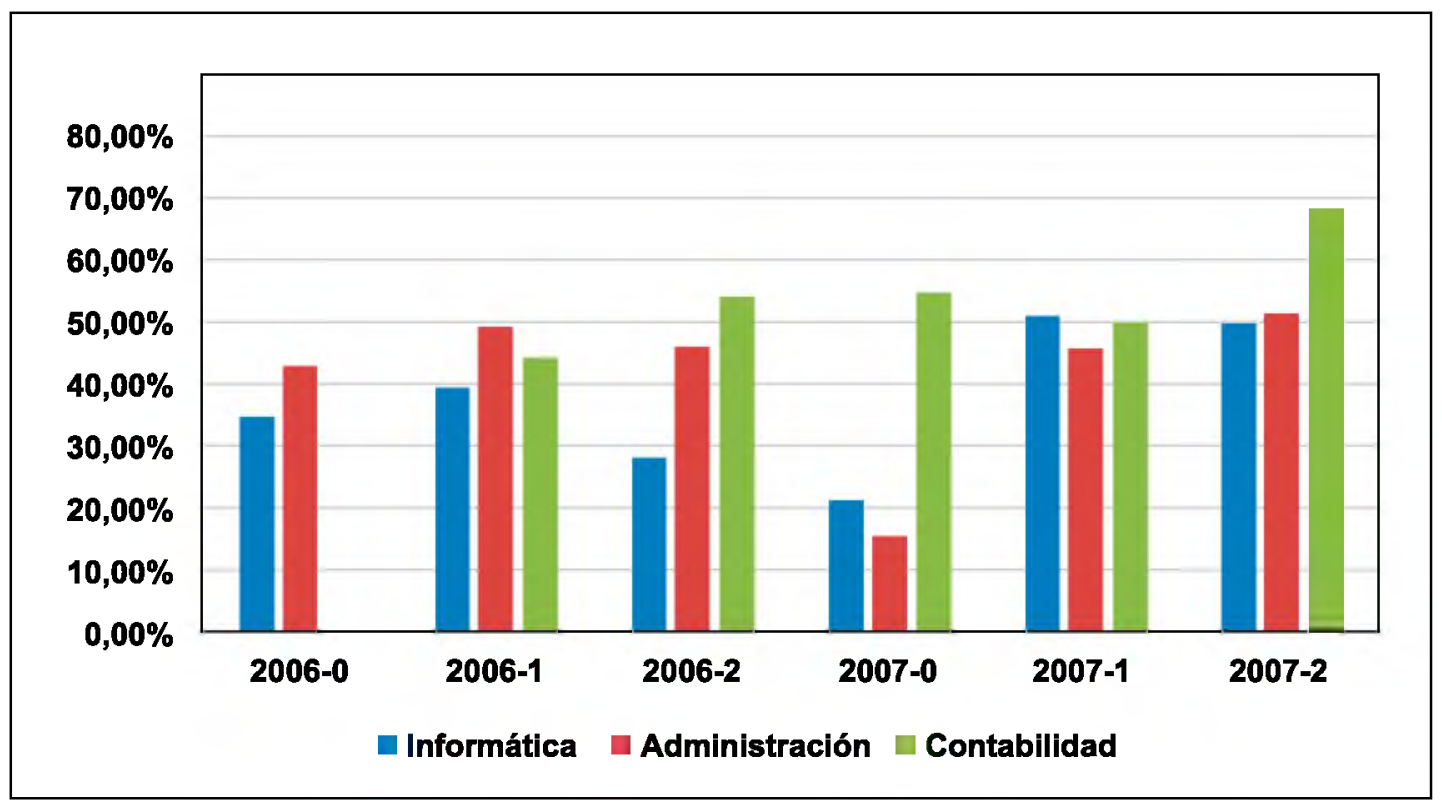

Figura $N^{\circ}$ 4: Incremento salarial por Escuela Profesional

tienen menos años de educación y mayor nivel de ingresos. Para esta estimación consideramos la variable sexo toma valores 1 para hombres y 0 para mujeres (figura $\mathrm{N}^{\circ}$ 3). Si bien no es crucialmente importante, creamos una variable nueva (genero01) que toma valores 0 para hombres y 1 para mujeres, se creó esta nueva variable para capturar en el modelo qué género gana más y por defecto hallamos que los hombres ganan más que las mujeres contrasta el comportamiento de nuestro mercado laboral.

En la figura $N^{\circ} 4$, se muestra el incremento salarial por carreras en los semestres de estudio, y observamos que la carrera que ha tenido el mayor crecimiento ha sido Contabilidad con un promedio de crecimiento del $54 \%$, seguida por la escuela de Administración con un promedio de crecimiento del $40 \%$; finalmente la escuela de Ingeniería Informática con un crecimiento promedio semestral del $37 \%$.

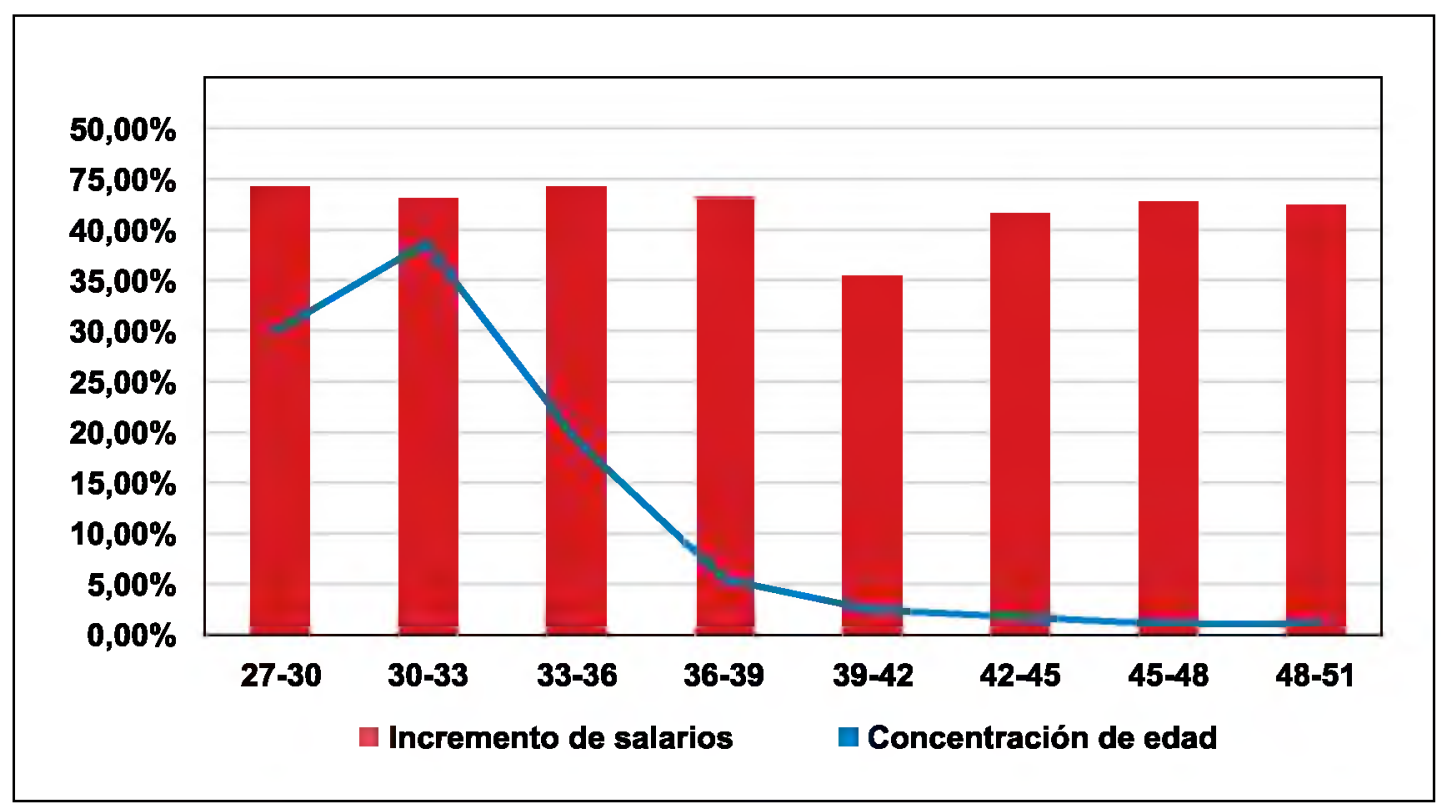

Figura $\mathrm{N}^{\circ}$ 5: Incremento salarial versus edad de egresados. 


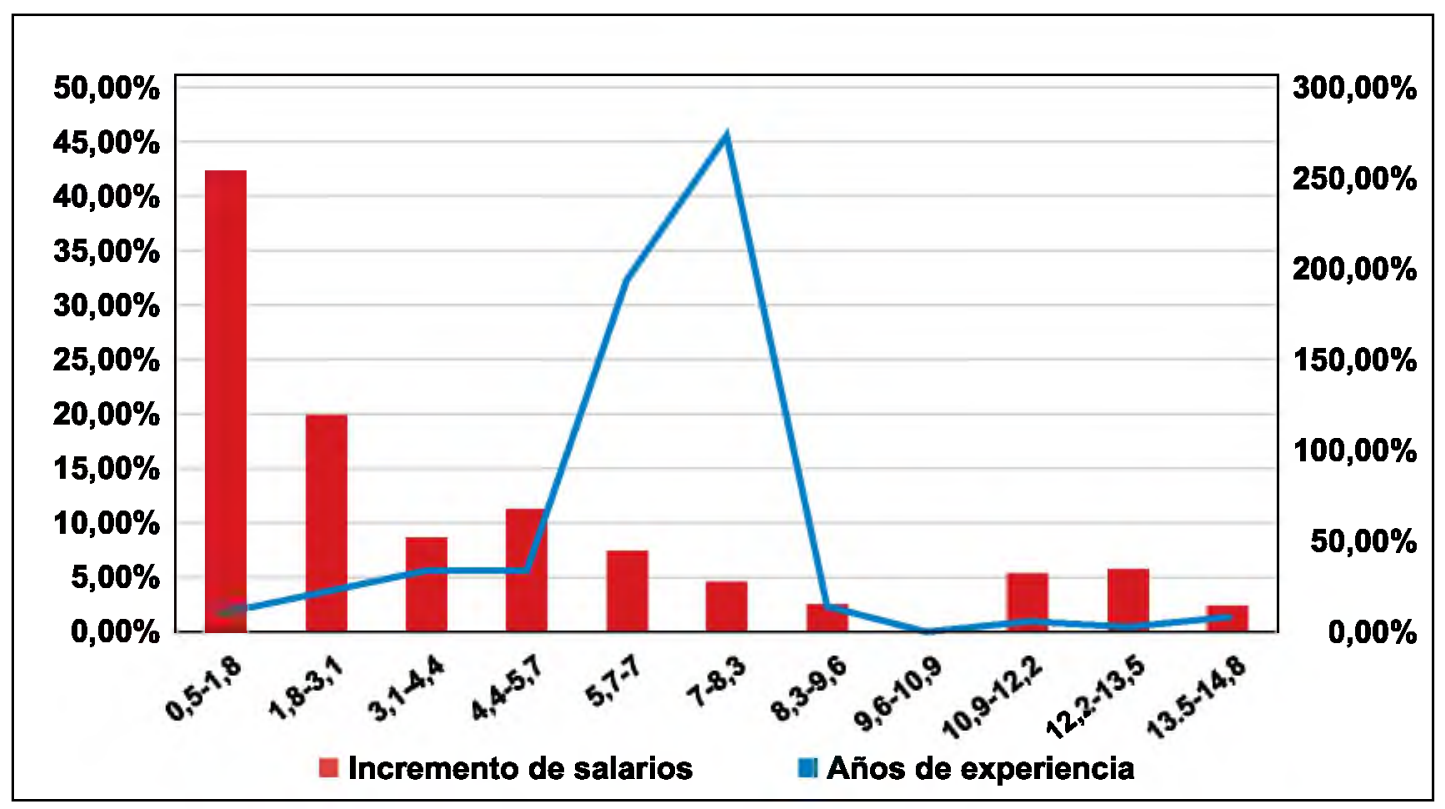

Figura $\mathrm{N}^{\circ}$ 6: Incremento salarial versus años de experiencia.

En la figura $N^{\circ} 5$, podemos observar que el incremento de los salarios de los egresados de la Universidad Continental es indirectamente a la edad de los egresados. Es decir a menos años de edad del egresado tiene mayor crecimiento en sus salarios. Esto también se explica porque pasando los 33 años los egresados ya van consolidándose en un centro laboral y la rotación laboral es menor.

En la figura $\mathrm{N}^{\circ} 6$, se observa la relación de incremento de salarios de los egresados es obtenido entre aquellos que tienen entre 4,4 años a 9,6 años de experiencia laboral.

\section{DISCUSIÓN}

Uno de los aspectos también a la hora de tomar en cuenta de por que el modelo tiene R2 muy bajo es que la Universidad Continental que empezó sus actividades con estas tres carreras, recién estaban siendo conocidos en el mercado laboral como egresados en comparación con los egresados de otras carreras por ejemplo, la variable experiencia si es significativa porque en los últimos años en el mercado laboral de nuestro país y de la región Junín es valorada para tener empleo y mejores niveles de ingresos.
Para la demostración de las hipótesis planteadas en la investigación se ha recorrido a la recopilación de datos a lo largo de los años considerados en el estudio; es bajo el valor obtenido con respecto al R2 no es fácil responder a esta pregunta, pues no hay problemas en las variables omitidas porque cuando aumentamos más variables el modelo mejoro.

Se determinó que existen diferencias en las tasas de rentabilidad educativa por sexo, el nivel de ingresos de los varones es mayor al de las mujeres. Además en los salarios existen diferencias entre contratos en base part o full time, de manera que deberían usarse variables que controlen por el número total de horas trabajadas.

Los resultados de la estimación de la ecuación de participación sugieren las siguientes conclusiones:

- Las variables determinantes de la rentabilidad de la inversión en educación superior de los egresados de la Universidad Continental son: nivel educativo alcanzado, salarios, experiencia laboral, edad y género. La variable que afecta negativamente al salario viene a ser la experiencia laboral y las variables que afectan positivamente al salario son edad y género. 
- Un año de educación adicional afecta negativamente a la probabilidad de participar en el mercado laboral, probablemente porque para una persona educada, es importante continuar con la educación. Esto está en consonancia con la teoría del capital humano y su visión de la educación como una inversión. La educación formal aumenta el salario de reserva y desalienta a las personas a trabajar.

- La experiencia incentiva a las personas para entrar en el mercado laboral, lo que es un resultado intuitivo. Además, la experiencia tiene un efecto positivo pero decreciente sobre la probabilidad de participar en el mercado laboral, lo que significa que tiene un máximo.

- Los egresados que han tenido mayor crecimiento salarial son de Contabilidad con un promedio de $54 \%$, seguido por los de Administración con un promedio $40 \%$ e Ingeniería Informática con $37 \%$ en promedio.

- La relación entre el incremento salarial versus la edad de los egresados es indirectamente proporcional. Es decir, a menos años de experiencia laboral mayor incremento salarial. Por otro lado, el mayor incremento salarial se logra entre los 4,4 años a 9,6 años de experiencia laboral.

- El análisis realizado en este trabajo, más allá de sus limitaciones, ofrece interesantes notas empírica sobre la relación entre la educación y la determinación de los ingresos. Además, el hecho de que el análisis es desarrollado por carreras, permite avanzar en las comparaciones entre ingresos de las escuelas profesionales de Ingeniería Informática, Contabilidad y Administración, aunque no puede soslayarse el hecho de que no se están considerando las amplias heterogeneidades existentes dentro de ellas.

\section{REFERENCIAS BIBLIOGRÁFICAS}

1. Mincer J. Schooling, Experience and Earnings. New York: Columbia University Press; 1974.

2. Bourguignon F, Ferreira F, Lusting $N$. The Microeconomics of Income Distribution Dynamies in East Asia and Latin America. Washington: World Bank and Oxford University Press; 2005.

3. Chiswick B. Interpreting the Coefficient of Schooling in the Human Capital Earnings Function. California: World Bank; 1997.

4. Angrist J, Krueger A. Does Compulsory Schooling Affect Schooling and Earnings? Quarterly Journal of Economics. 1991: 106(4): 979-1014.

5. Lucas R. On the Mechanics of Developement Planning. Journal of Monetary Economics. 1988; 22: 3-42.

6. Heckman J. Sample Selection Bias as a Specification Error. Econometrica. 1979; 47(1): 153-161.

7. Yamada G. Retornos a la Educación Superior en el Mercado Laboral. Lima: CIUP; 2007. 